\title{
Gravity wave initiation of equatorial spread F/plasma bubble irregularities based on observational data from the SpreadFEx campaign
}

\author{
M. A. Abdu ${ }^{1}$, E. Alam Kherani ${ }^{1}$, I. S. Batista ${ }^{1}$, E. R. de Paula ${ }^{1}$, D. C. Fritts ${ }^{2}$, and J. H. A. Sobral ${ }^{1}$ \\ ${ }^{1}$ Instituto Nacional de Pesquisais Espaciais, Sao Jose dos Campos, SP, Brazil \\ ${ }^{2}$ NorthWest Research Associates, Colorado Research Associates Division, Boulder, USA
}

Received: 23 April 2008 - Revised: 6 January 2009 - Accepted: 13 January 2009 - Published: 1 July 2009

\begin{abstract}
The data from ground based experiments conducted during the 2005 SpreadFEx campaign in Brazil are used, with the help of theoretical model calculations, to investigate the precursor conditions, and especially, the role of gravity waves, in the instability initiation leading to equatorial spread F development. Data from a digisonde and a $30 \mathrm{MHz}$ coherent back-scatter radar operated at an equatorial site, Sao Luis (dip angle: $2.7^{\circ}$ ) and from a digisonde operated at another equatorial site (dip angle: $-11.5^{\circ}$ ) are analyzed during selected days representative of differing precursor conditions of the evening prereversal vertical drift, F layer bottom-side density gradients and density perturbations due to gravity waves. It is found that radar irregularity plumes indicative of topside bubbles, can be generated for precursor vertical drift velocities exceeding $30 \mathrm{~m} / \mathrm{s}$ even when the precursor GW induced density oscillations are marginally detectable by the digisonde. For drift velocities $\leq 20 \mathrm{~m} / \mathrm{s}$ the presence of precursor gravity waves of detectable intensity is found to be a necessary condition for spread F instability initiation. Theoretical model calculations show that the zonal polarization electric field in an instability development, even as judged from its linear growth phase, can be significantly enhanced under the action of perturbation winds from gravity waves. Comparison of the observational results with the theoretical model calculations provides evidence for gravity wave seeding of equatorial spread $\mathrm{F}$.
\end{abstract}

Keywords. Ionosphere (Ionospheric irregularities; Plasma waves and instabilities)

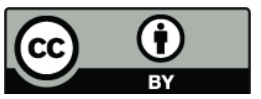

Correspondence to: M. A. Abdu (maabdu@dae.inpe.br)

\section{Introduction}

Equatorial spread $\mathrm{F}$ post-dusk development occurs under electrodynamical processes unique to the sunset transition in the E- and F layers of the ionosphere. It is well known that equatorial $\mathrm{F}$ layer rises up under the action of an enhanced evening eastward electric field (known as the prereversal enhancement in the zonal electric field - PRE) causing the bottom-side density gradient region of the rising layer to become unstable to density perturbations leading to the spread F/plasma bubble irregularity (ESF) development. The Rayleigh-Taylor interchange instability (RTI), or the generalized gradient drift instability (GDI), is believed to be the mechanism by which an instability initiated at the F layer bottomside develops into flux tube aligned plasma depletions (with their cascading irregularity structures) rising to the topside of the $\mathrm{F}$ region. The signatures of these plasma irregularities have wide ranging features as registered by radars, digisondes, optical imagers, GPS receivers and space-borne detectors. ESF presents a large degree of variability at medium term (seasonal), long term (solar cycle) and short term (day-to-day) scales. The last of these is the least understood aspect and constitutes the most challenging problem for present day ESF investigations. In this respect it is of fundamental importance to evaluate in detail the precursor conditions of the ambient IT (IonosphereThermosphere) conducive or otherwise to ESF development. The most well-known and so far well-documented precursor conditions concern the post sunset $\mathrm{F}$ layer height increase due to the PRE arising from the sunset electrodynamic coupling processes in which the thermospheric zonal wind (eastward in the evening) and the decaying E layer conductivity (presenting itself as longitudinal/local time conductivity gradient across the sunset terminator) are the most important control parameters (Rishbeth, 1971; Farley et al., 1986). A

Published by Copernicus Publications on behalf of the European Geosciences Union. 
large eastward thermospheric wind in the evening could produce large vertical drift and hence the $\mathrm{F}$ layer rise to higher levels thereby enhancing significantly the spread F instability growth rate by Rayleigh-Taylor instability mechanism. The requirements on the PRE/evening vertical drift and the height of the F layer for the spread F "on" and "off" conditions and its varying growth rates and intensities have been widely discussed in the literature (e.g., Farley et al., 1970; Abdu et al., 1983, 2006; Fejer et al., 1999; Sultan, 1996; Sastri et al., 1997). Models have been developed to investigate the effects of the evening zonal electric fields, F layer bottom-side density gradients, and zonal, meridional and vertical winds on the ESF instability development conditions (e.g., Zalesak et al., 1982; Sekar et al., 1994; Maruyama, 1988; Keskinen et al., 2003; Kudeki et al., 2007; Kherani et al., 2009a). An important prerequisite concerns the nature and the intensity of the density perturbation needed to initiate the instability growth, which appears to be the least understood aspect of the ESF processes. Although certain threshold values for the PRE and the F layer heights for ESF development can be established statistically for a given solar flux, season, or longitude, such threshold conditions are not directly verifiable in specific cases studies, the reason being the possible role of a precursor density perturbations in the form of a wave disturbance with variable intensity and wavelength needed to initiate the instability process. Observational data shows that spread F invariably develops following the occurrence of sufficiently large evening vertical drifts, whether due to the normal (quiet time) evening PRE arising from $F$ layer dynamo or due to magnetic storm associated penetrating electric fields (Abdu et al., 2003). This situation would point to (a) the need for a seed perturbation to be always present in the IT in the form of geophysical noise (e.g., Hanson et al., 1986) or (b) a seed mechanism arising from the very process of the layer uplift that necessarily (and nearly always) precedes ESF. Since we have no clear evidence for the existence of the latter possibility, we need to consider the need for seed perturbations in the form of geophysical noise that includes the atmospheric gravity wave spectrum. The gravity wave wind perturbations could produce density modulation (possibly from meridional wind component) as well as generate polarization electric field (from the zonal and vertical wind components) contributing to the instability growth. The gravity wave air motion being perpendicular to the propagation wave vector, we could expect that waves propagating upward at slant angles in general could provide the required perturbation winds leading to density perturbation as well as polarization electric fields. It looks possible that all possible modes of gravity wave propagation could in some way contribute to the instability growth under right conditions of the ambient ionosphere. Numerical simulations studies have shown that density perturbations of the order of $5 \%$ or even less, attributed to gravity waves, can initiate instability growth leading to vertical development of bubbles to topside ionosphere under typical dynamic state of the background iono- sphere of the sunset period (e.g., Zalesak et al., 1982; Keskinen et al., 2003). Non linear evolution of equatorial spread $\mathrm{F}$ from gravity wave seeding has been investigated in detail by Huang and Kelley (1996a, b). Observational evidence of a possible association between gravity waves and ESF has been suggested in statistical data comparisons by several authors (see for example Rottger, 1981; McClure et al., 1998; Abdu, 2001). Gravity wave generation in tropospheric convective regions and the upward propagation of the wave at slant angles to ionospheric height have been suggested as a possible cause of such association (see for a detailed evaluation, Fritts et al., 2008). Also, production of electric field perturbations by gravity wave winds in the $\mathrm{E}$ region has been proposed to be a plausible mechanism for initiating spread F (Prakash, 1999). Independent of the sources of the gravity waves, however, case studies of gravity wave modulation of radar irregularity (3-m) plume formation, over Jicamarca, have been analyzed by Kelley et al. (1981) and Hysell et al. (1990). In particular, for the spectacular gravity wave events studied by these authors the large scale undulations of the bottom-side F layer and the irregularity plumes rising vertically from the descending side of the undulations suggested the role of gravity waves in modulating the RT instability growth conditions. Gravity wave modulations of well developed radar irregularity $(5-\mathrm{m})$ plume formations and $\mathrm{F}$ layer electron density contours have been observed rather frequently over Sao Luis, although such results have not so far been published. On the other hand, large scale modulations in F layer heights/densities induced by gravity waves as diagnosed in ionograms did not lead to development of spread F over Cachoeira Paulista during pre sunrise hours (Abdu et al., 1982).

The presently challenging task, however, is the identification of gravity waves as a precursor condition for the post dusk ESF development. This would necessarily require the detection of these waves during local times immediately preceding a spread $\mathrm{F}$ onset and their being pursued, possibly with enhanced amplitudes, into night hours of spread $\mathrm{F}$ activity. The Spread F Experiment (SpreadFEx) campaign had as its main objective, as explained by Fritts et al. (2008), the identification of a cause-effect sequence for the ESF development that would involve a source of gravity wave generation in regions of deep tropospheric convection and the slant angle/upward propagation of these waves through the mesosphere, the thermosphere and into the bottom-side $\mathrm{F}$ layer where the electron density perturbation and polarization electric fields induced by these waves could initiate the spread $\mathrm{F}$ instability process. In this paper we show that, under the conditions favorable for the upward propagation of GWs reaching thermospheric heights that characterized the SpreadFEx campaign epoch as explained in the paper by Fritts et al. (2008), electron density perturbations presenting clear GW characteristics are indeed observed at the bottom side of the equatorial $\mathrm{F}$ region. We will show that these waves were precursor to the post dusk spread $\mathrm{F}$ that developed over 
Sao Luis whose variable intensity appears to depend upon the characteristics of the precursor GWs. Using theory based model calculations on the linear growth rate of the GW induced instability electric fields we will show that precursor gravity waves having the observed characteristics are indeed necessary to explain the relationship of these waves with the observed spread $\mathrm{F}$ characteristics.

Complementary or alternative mechanisms for the ESF seeding process have been proposed recently that are based on wind driven instabilities. This requires horizontal density gradients associated with $\mathrm{F}$ region bottom-side undulations in the presence of a zonal wind in the plasma reference frame. The basic instability growth rate as obtained by Linson and Workman (1970) was applied by MacDougall et al. (1998) to explain growth rates of medium scale $(\mathrm{km}$ size) spread $\mathrm{F}$ irregularities of post midnight/pre sunrise hours observed by ionosonde over Fortaleza. In the evening sector the zonal thermospheric wind is known to accelerate eastward, which therefore becomes more effective (for instability growth) in the reference frame of a non drifting or westward drifting plasma (of the F layer bottom side) being part of the wellknown post sunset plasma flow vortex system (Kudeki and Bhattacharyya, 1999). From stability analysis of a realistic post sunset F region, Kudeki et al. (2007) obtained for this case large growth rate, (for local as well as non local conditions) for intermediate scale instability development (the scale size being comparable to the F layer bottom-side gradient scale length). Due to its rather fast growth rate (of several e-folds per hour) it was proposed to be an adequate mechanism for the generation and maintenance of the more frequently observed bottom-type structures that could serve as seed perturbations so that, when the conditions evolve into sufficiently large RTI growth rates, it could lead to development of vertically rising bubble structures. This mechanism has a scale size dependent growth rate, favoring shorter wavelengths, that is, $\lambda<l_{o}$ ( $\lambda$ being the scale size in the zonal direction and $l_{o}$ the $\mathrm{F}$ flayer bottomside scale length which typically is of the order of $20-30 \mathrm{~km}$ ). The growth rate decreases significantly for larger scale sizes as shown by Kudeki et al. (2007). The scale size of the gravity waves as observed by digisonde in the example to be presented here are of the order of $200-300 \mathrm{~km}$. The scale sizes of the mesospheric gravity waves and of the bubbles from airglow imager data over Cariri during the SpreadFEx campaign as analyzed by Takahashi et al. (2009) are typically in excess of $100 \mathrm{~km}$. According to Kudeki et al. (2007) the wind driven instability growth rate for these scale sizes is significantly smaller, but still could compete with the growth rate due to $\boldsymbol{E} \times \boldsymbol{B}$ uplift. Kudeki et al. (2007) have argued that for sufficiently large zonal winds, of the order of $200 \mathrm{~m} / \mathrm{s}$, "the initial seeding amplitude of the irregularities set by external factors such as gravity waves should not be a too critical of an input to the overall process". The large zonal wind could also produce large PRE, that is, large vertical drift, enhancing the instability growth by the R-T mechanism. In the present in- vestigation we have focused on spread $\mathrm{F}$ instability development under relatively smaller PRE intensity that should require the presence of relatively smaller zonal winds in the evening. For example the PRE amplitudes in our case studies (to be presented below) vary from 15 to $30 \mathrm{~m} / \mathrm{s}$ for which the zonal wind velocities vary around $100 \mathrm{~m} / \mathrm{s}$ or less (see, Abdu et al., 1995). This relationship between the zonal wind and the PRE was obtained using an E- F- region electrical coupling model by Batista et al. (1986) that was based on the formalism by Heelis et al. (1974). Under the relatively low zonal winds, representative of the cases analyzed here, the initiation of bubble irregularities of the scales sizes discussed here would appears to be less likely operating through a process dominated by wind driven instability mechanism, which may still be necessary for the maintenance of the bottom-side irregularities. Also, at a later phase of the significant westward plasma flow (of the bottom-side) Hysell and Kudeki (2004) obtained a smaller growth rate by their shear instability mechanism for similar scale sizes which they also suggested as a possible seed for eventual bubbles development. Implicit in these mechanisms is the requirement for a pre existing undulation in the $\mathrm{F}$ layer bottom side density distribution, which must be induced most likely by gravity waves. The characteristics of the gravity waves (such as, amplitude, wavelength, frequency, propagation direction, etc.) present in the evening sector IT appear to determine the nature of the F layer bottom side density undulations, and hence the relative importance of the different possible mechanisms at play in the spread F/bubble development process, that could vary from one day to the other. In the following we will present an analysis of gravity wave signatures in the $\mathrm{F}$ layer bottom side densities and their possible impact on the varying intensities of post dusk spread $\mathrm{F}$ structuring as observed during the SpreadFEx campaign.

\section{Experimental data}

The experimental data analyzed here were collected during the SpreadFEx campaign by a set of instruments consisting of a digisonde and a $30 \mathrm{MHz}$ coherent back-scatter radar installed at Sao Luis $\left(44.2^{\circ} \mathrm{W}, 2.33^{\circ} \mathrm{S}\right.$, dip angle: $\left.-2.7^{\circ}\right)$, and another digisonde, a digital portable sounder (DPS-4), operated at Fortaleza $\left(38.45^{\circ} \mathrm{W}, 3.9^{\circ} \mathrm{S}\right.$, dip angle: $\left.-11.5^{\circ}\right)$. The RTI map obtained by the VHF radar provided information on the 5-m irregularity structures, representing spread F evolution, at 1-mi resolution. Data collection by the digisonde was at 10-min cadence. The SAO explorer software of the digisonde produced the $\mathrm{F}$ region true heights that were used to calculate the vertical drift velocities as $d\left(h_{F}\right) / \mathrm{dt}$. The ionogram inversion algorithm used in the SAO explorer software for the automatic real height calculation has been described by Huang and Reinisch (1996). The true heights so obtained have been validated by comparison with the electron density profiles measured by the incoherent scatter radar 
over Millstone Hill, obtaining excellent agreement. Also, good agreement has been found between the vertical drift calculated from the true heights as $d\left(h_{F}\right) / \mathrm{dt}$ and that obtained from Doppler data (Abdu et al., 2006b) using the digisonde drift explorer software (Scali et al., 1995) as well as that measured by the Jicamarca incoherent scatter radar (Bertoni et al., 2006). The true heights were used also to extract the gravity wave oscillations at specific plasma frequencies (as explained later). At this point it is appropriate to mention that ionosonde method has been used in reliable ways to detect and quantify gravity wave effects in the low latitude ionosphere (see for example, Abdu et al., 1982, and references therein). The present study represents the first attempt to employ such method for evaluating the gravity wave effects as precursor to the post sunset initiation of equatorial spread F. We will therefore comment below on the reliability of the method for detecting the presence of precursor GWs which necessarily demands more rigorous sensitivity considerations.

The digisonde transmits coded pulses at digital frequencies (nominally 200 frequencies) scanning from 1 to $20 \mathrm{MHz}$ that are reflected from the ionosphere at plasma frequencies $f_{p}<f o \mathrm{~F} 2$. The scale size of the reflecting region corresponds to that of the first Fresnel zone, which for example is $7.5 \mathrm{~km}$, at $7 \mathrm{MHz}$ reflected from a height of $350 \mathrm{~km}$. The resolution in the electron densities measured at the frequencies spaced at $100 \mathrm{kHz}$ is of the order of 3-4 percent of the ambient densities (for $f_{p}$ in the region of 5 to $10 \mathrm{MHz}$, for example). The height resolution for the virtual heights $\left(h^{\prime} F\right)$ is determined by the pulse width/coding and varies from $5 \mathrm{~km}$ to $10 \mathrm{~km}$ for the height ranges of $500 \mathrm{~km}$ to $1000 \mathrm{~km}$ that are usually set for the ionograms. The true heights that are extracted from a series of virtual heights (by the ionogram inversion method mentioned before) also have similar degree of height resolution as that of the virtual heights. The true heights are used for calculating the parameters of our interest here, such as the scale length of the bottomside density distribution $\left(l_{o}=n / d n / d h F\right)$, the vertical drift $\left(d h_{F} / d t\right)$ and the height oscillations due to GWs. None of these parameters suffer the limitations imposed by the above mentioned height resolutions since we are concerned with relative variations in the heights at different plasma frequencies, or at different time steps corresponding to the observational cadence $(10 \mathrm{~min}$ in the present data set). Oscillations in true heights at adjacent plasma frequencies prior to sunset could be noted in Figs. 1, 2 and 3 (as to be explained later) to have periodicities of the order of one hour, with oscillation amplitudes as low as $5 \mathrm{~km}$ or less, presenting downward phase propagation characterizing themselves to be GW signatures. A height oscillation by $5 \mathrm{~km}$ corresponds to 20 percent modulation in electron density assuming a bottom side scale length of $30 \mathrm{~km}$ as an example. Such amplitudes of density fluctuation are compatible with the predictions for $\mathrm{F}$ layer bottomside density perturbations produced by upward propagating GWs from tropospheric sources under nominal background atmospheric and ionospheric conditions (see Fritts et al., 2008). While the lower limit of the GW period detected by the digisonde is dependent on the observational cadence, that of the density perturbations is dependent on the operational settings of the instrument. At the settings used in the data presented here height oscillations of $2-3 \mathrm{~km}$ corresponding to density perturbations of 6-10 percent can be diagnosed. This point needs to be studied further in detail, however.

\section{Results}

\subsection{Signatures of gravity waves and evening prereversal electric field in $F$ layer heights}

For an assessment of gravity wave (GW) influences on spread $\mathrm{F}$ instability initiation it is important to examine any wave fluctuations in electron density immediately preceding an ESF event so that possible influences from their continuing presence on instability growth can be evaluated on the basis of the ESF development that may follow. Therefore identification of GW presence in the IT during pre sunset hours has fundamental importance for evaluating the role of such GWs in the post dusk ESF. Both horizontal and vertical components of GW winds can influence instability growth rates. The different ways in which GWs at $\mathrm{F}$ layer heights can influence the spread $\mathrm{F}$ development can be stated as follows:

1. Modulation of the dusk sector F layer heights through large undulations in electron density iso-lines whereby the elevated bottom-side density gradient regions will become unstable to GRTI (generalized Rayleigh-Taylor instability) with large instability growth rates, an example of which appears to be present in GW event (observed over Jicamarca radar) analyzed by Kelley et al. (1981);

2. Modulation of the PRE by having its peak intensity enhanced by in-phase superposition of zonal (eastward) winds from the background thermospheric tidal source and perturbation zonal winds due to GWs. It is wellknown that the background thermospheric zonal wind that turns eastward in the evening is a major driver of the PRE (e.g., Rishbeth, 1971; Heelis et al., 1974; Farley et al., 1986; Batista et al., 1986). Such an increase of the PRE amplitude can contribute to an enhanced GDI (gradient drift instability) growth rate factor $\left(V_{y} / l_{o}\right)$ besides raising the $\mathrm{F}$ layer bottom-side to larger heights where the gravitational RTI growth rate factor $\left(g / v_{i} l_{o}\right)$ is enhanced as well. The zonal perturbation velocity of the GW at sunset time could also contribute a factor to the instability growth (in addition to the $V_{y} / l_{o}$ factor) on the line discussed by Kudeki et al. (2007);

3. Direct seeding of the instability by GWs providing the density perturbations/ undulations and the development 
of the polarization electric fields that grow with the density perturbations, constituting the instability growth process. The GW zonal and vertical winds contribute directly to the polarization electric field that feeds the instability growth. (The wind driven instability processes discussed earlier could also belong to this type). The GWs of this item are generally of smaller amplitude (than, especially, those of item-1 above) and are typically representative of the cases to be discussed here. Their characteristics (periods, wavelengths. etc. to be discussed below) appear to be consistent with those of the GWs present in the $\mathrm{F}$ region bottom-side after having propagated possibly from the tropospheric convective sources as described in the paper by Fritts et al. (2008).

GW zonal and vertical wind components in the equatorial $\mathrm{F}$ region could generate dynamo electric fields that will drive currents closing through the conjugate $\mathrm{E}$ layers connected by magnetic field lines. In the presence of non conducting $\mathrm{E}$ layers typical of the night conditions, no current flow occurs, resulting in buildup of polarization electric fields in the $\mathrm{F}$ region according to the relation: $\Sigma_{P}\left(E+\delta W \times B_{o}\right)=0$, where $\boldsymbol{E}$ is the polarization electric field, $\Sigma_{P}$ is the Pedersen conductivity, $\delta W$ is the gravity wave wind, and $B_{o}$ is the Earth's magnetic field. The polarization electric field causes the plasma to move with the neutral wind of the GW. In this way F layer height oscillations induced by GWs can be detected under night time conditions. During day time the large E layer conductivity permits current flow causing the shorting of the polarization electric field leading to negligible GW effect at $\mathrm{F}$ layer heights, the GW effect gaining amplitude as the E layer conductivity decays with sunset. On the other hand any GW oscillations in meridional winds that are undetectable very close to the dip equator are amenable to detection as $\mathrm{F}$ layer height oscillations at a location with inclined magnetic field. Thus such height displacements, expected to be negligible over Sao Luis (being closer to the dip equator with dip angle: $-2.7^{\circ}$ ), are detectable over Fortaleza (with dip angle: $-11.5^{\circ}$ ) during both day and night, whereas any dynamo effect from zonal/vertical winds will be detectable only during night hours at both sites. For this reason, we have used the $\mathrm{F}$ layer height oscillations over Fortaleza to assess the presence of GWs during pre sunset extending to night hours, and to investigate their possible influence on the spread F development conditions over Sao Luis, assuming that nearly identical GWs perturbations exist over the two sites. Such an assumption is justifiable because (1) the zonally propagating gravity waves are the more likely ones to be present in the bottomside F layer (Fritts et al., 2008) and (2) Sao Luis and Fortaleza are separated more in longitude than in latitude and therefore there appears to be no reason for a zonally propagating GW not to be presenting the same characteristics at these two locations. We note further that while at an off-equatorial location (like Fortaleza) the perturbation meridional winds of the GWs produces height oscillations, representing the density perturbation, that can be detected as a precursor to spread $F$, the effect of the zonal and vertical wind perturbations of the same GW can be diagnosed over the dip equator (represented by Sao Luis in our case) only after the spread $\mathrm{F}$ irregularities begin to evolve.

In the left panel of Fig. 1 are shown the band-pass filtered oscillations in $\mathrm{F}$ layer true heights $\left(h_{F}\right)$ as observed by the digisonde at specific plasma frequencies $(5,6,7$, and $8 \mathrm{MHz})$ over Fortaleza, plotted from 15:00 LT (18:00 UT) to midnight (27:00 UT) for three days as examples of GW manifestations during the pre and post dusk hours. The band-pass filtered oscillations include periods from 20-min to 3-h only. They are plotted with their base values displaced by $5 \mathrm{~km}$ (cumulatively) at each frequency. The dominant oscillations have periods of the order of an hour and they present downward phase propagation rather clearly during pre dusk hours. The inferred characteristics of these waves, such as the wavelength, period and perturbation wind velocities appear to be compatible with the corresponding values estimated for GWs propagating from tropospheric convective regions to this height region, as evaluated by Fritts et al. (2008). As an example, we consider the $\mathrm{F}$ layer height perturbations of 5 October in Fig. 1 (top panel). Here during the hours just prior to sunset the downward phase propagation suggests a rather well defined vertical half wavelength $\left(\lambda_{y} / 2\right)$ for the wave (as judged from the nearly opposite phases of 5 and $8 \mathrm{MHz}$ oscillations, noted at 20:00 UT/17:00 LT). Using the difference in true heights at these frequencies $\left(\Delta h_{F(8-5 \mathrm{MHz})}\right)$ we can determine the $\lambda_{y}$ to be $50 \mathrm{~km}$. The horizontal wavelength $\lambda_{x}$ can be determined from the relation $\omega^{2} \lambda_{y}^{2}=\left(N^{2}-\omega^{2}\right) \lambda_{x}^{2}$, where $N$ is the buoyancy frequency and $\omega$ is the wave frequency (Hines, 1960). Using the buoyancy period to be $11 \mathrm{~min}$ at heights above $200 \mathrm{~km}$ for medium solar conditions (Fritts et al., 2008), and the wave period to be $60 \mathrm{~min}$. we obtain a horizontal wavelength of $270 \mathrm{~km}$ and a horizontal velocity of $75 \mathrm{~m} / \mathrm{s}$. These values are compatible with the parameters predicted by Fritts et al. (2008) (see, for example, the values in their Table 4) at $\mathrm{F}$ layer bottom-side heights for upward propagating tropospheric GWs diagnosed under the SpreadFEx campaign conditions. The oscillation amplitude varies significantly from one day to another. The dominant wavelength appears to be also variable on a day-to-day basis. We note that the downward phase propagation characteristics (in Fig. 1) become less evident during post dusk hours. This may in part be due to the fact that the electric fields arising from the action of the GW winds and/or that associated with the instability development seeded by these waves could also influence the height oscillations, as will be seen from comparison with the spread F data (to be discussed later).

In the right panel are shown examples of vertical drifts $\left(V_{y}\right.$ marked as $V z$ in Figs. 1, 2 and 3) derived as $d\left(h_{F}\right) / \mathrm{dt}$ for the same set of days. Drifts calculated at four plasma frequencies $(5,6,7$ and $8 \mathrm{MHz})$ are shown and they lie close to each other and to their mean curve also shown. The main point to 

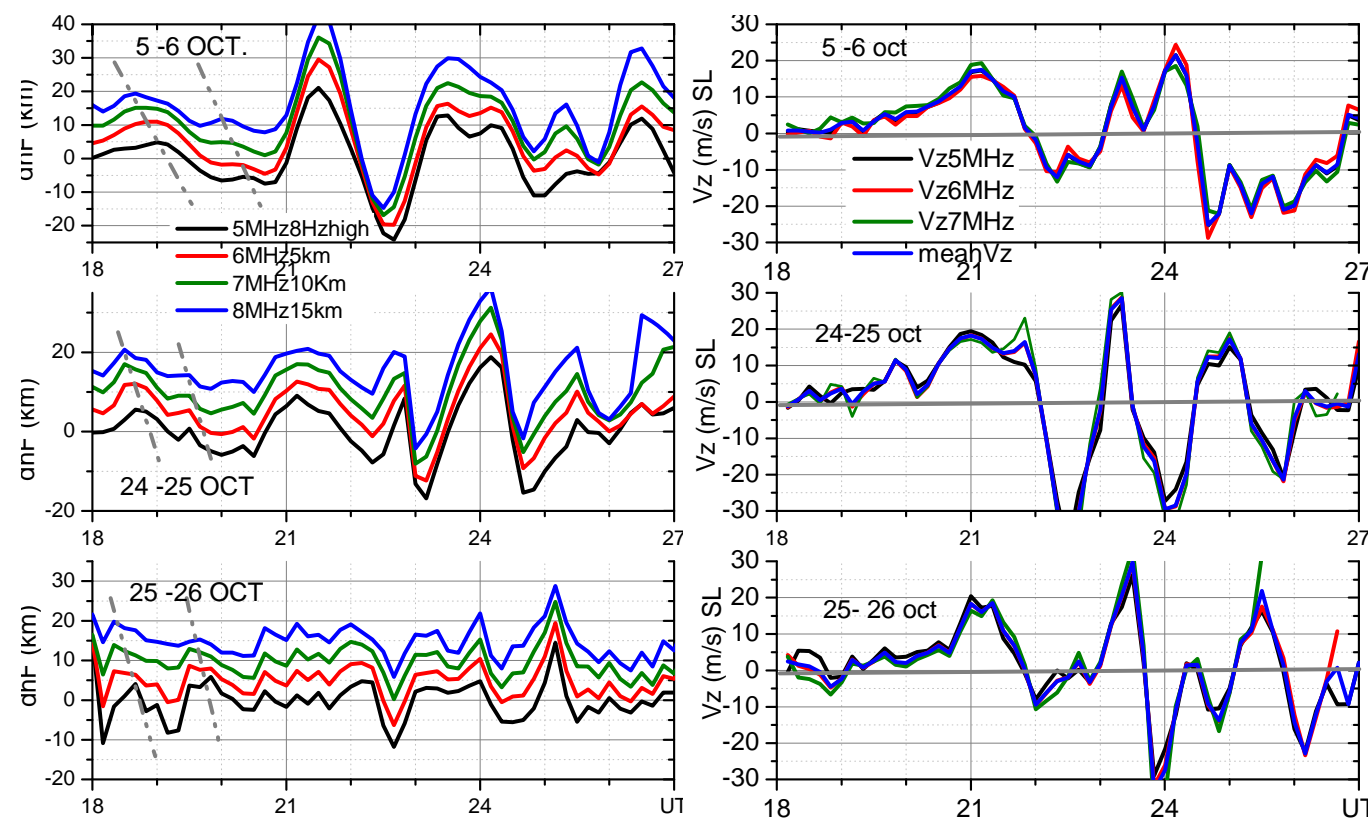

Fig. 1. In the left panels are shown perturbations in F layer true heights at frequencies: 5, 6, 7 and $8 \mathrm{MHz}$, band-pass filtered between periods $20 \mathrm{~min}$ and $3 \mathrm{~h}$. The results are shown for three days. The plots are displaced cumulatively by $5 \mathrm{~km}$ at each frequency, and are in $\mathrm{UT}(=\mathrm{LT}+3 \mathrm{~h})$. We can clearly identify the presence of gravity waves from their downward phase propagation during the period immediately before the sunset (21:00 UT/18:00 LT). In the right panels are shown for the same three days the vertical drifts calculated as $d\left(h_{F}\right) / \mathrm{dt}$. The peak amplitude of the evening prereversal drift enhancement occurs near 21:00 UT/18:00 LT. Much of the drifts at later hours are influenced by range spreading due to ESF irregularities.

note in these plots is the evening enhancements in $V_{y}$ (near 21:00 UT) that correspond to the prereversal enhancement in zonal electric field (PRE), an important prerequisite (perhaps the most important one depending upon its amplitude) for the initiations of ESF development. It has been noted from statistical analysis of the data in the Brazilian sector that an evening vertical drift in excess of $38 \mathrm{~m} / \mathrm{s}$ (on an average) is required as a precondition for the occurrence of well extended ( $>1000 \mathrm{~km}$ above equator) topside bubbles (e.g., Abdu et al., 2006). We note in Fig. 1 larger amplitudes of the fluctuations in the vertical drift during later hours (usually after 22:00 UT in these cases) that must have been influenced by the dynamics of the developing bubbles present at these times. It is important to point out here that under the low solar activity conditions (F10.7: 75) of the SpreadFEx campaign, the PRE vertical drifts are generally small (e.g., Fejer et al., 1991). The peak velocities of the PRE (the $V_{y p}$, occurring at 18:00 LT/21:00 UT), that is, $18 \mathrm{~m} / \mathrm{s}$ in the examples in Fig. 1, are considered to be insufficient (or marginal) for well extended bubble development (Abdu et al., 1983, 2006; Fejer et al., 1999), and hence they appear to be somewhat ideal for evaluating any possible contribution to the ESF generation arising from GW effects.

\subsection{Complementary roles of GWs and PRE in spread F development: some case studies}

Figure 2a presents in the top panel the RTI plots of the 5-m irregularity distribution from the $30 \mathrm{MHz}$ radar over Sao Luis on the night of 23-24 October. The F layer true heights for specific plasma frequencies at $1 \mathrm{MHz}$ interval, starting with $5 \mathrm{MHz}$ are shown in the second panel. The vertical drifts taken as the averages of the drifts at four plasma frequencies, 5, 6, 7, and $8 \mathrm{MHz}$, are shown in the lower (third) panel. Also shown in this figure (fourth panel) are the band-pass filtered oscillations in the F layer true heights at 5, 6, 7 and $8 \mathrm{MHz}$ (over Fortaleza) similar to the plots in Fig. 1. In the bottom panel we have presented the R-T growth rate factor, $V_{y} / l_{o}$, corresponding to the vertical drift for this case, where $l_{o}$ is the F layer bottom-side density gradient as measured between the plasma frequencies $5 \mathrm{MHz}$ and $7 \mathrm{MHz}$. It may be commented that while the RTI map (indicating spread F activity) starts just around 18:30 LT (21:30 UT) the other parameters are shown starting at 15:00 LT to be able to evaluate the spread $\mathrm{F}$ precursor conditions of these parameters. The PRE drift velocity (on 23 October) attained $32 \mathrm{~m} / \mathrm{s}$ near 21:40 UT (18:40 LT) that produced a growth rate factor of $5 \times 10^{-4} \mathrm{~s}^{-1}$. The corresponding instability growth time is $33 \mathrm{~min}$ and we note the onset of $5-\mathrm{m}$ irregularities just after 22:10 UT (19:10 LT) in the top panel, which is to be expected 




Fig. 2a. Plots for the 23-24 October 2005. RTI map of 5-m irregularity distribution as observed by the $30 \mathrm{MHz}$ radar over Sao Luis (top panel); F layer true heights at plasma frequencies 5, 6, 7 and $8 \mathrm{MHz}$ (panel 2 from top); Mean of the drift velocities calculated as $\mathrm{d}(\mathrm{hF}) / \mathrm{dt}$ at frequencies 5, 6, 7, and $8 \mathrm{MHz}$ (panel 3 from top); Band-pass filtered (for 20-min. to 3-h periods) height oscillations at frequencies 5, 6, 7, and $8 \mathrm{MHz}$ (panel 4 from top); Instability growth rate due to the term $V z / L$ of the instability linear growth rate factor (bottom panel). The " $V z$ " in this and other figures is the same as the " $V_{y}$ " in the text.

if plasma depletion was already evolving by this time. Since the irregularity developed after a growth time corresponding to a single e-fold there appears to be present a seeding GWs perturbation though perhaps of weak enough intensity to be marginally in the detection limit of the digisonde on this evening. The spread F onset seems to concur with the drift reversal to downward that occurred at 22:00 UT/19:00 LT. The rapid bubble growth into topside $5-\mathrm{m}$ plume structure seems to have been helped by the large $\mathrm{F}$ layer height resulting from the rather large vertical drift (in other words, contribution from the R-T growth factor due to $\mathrm{g} / \mathrm{v}_{i n} l_{o}$ term seems to have contributed to a faster growth rate of the bubble on this evening). It is important to note (in panel 4 of Fig. 2a) that on this evening the perturbations in $\mathrm{F}$ layer heights just prior to

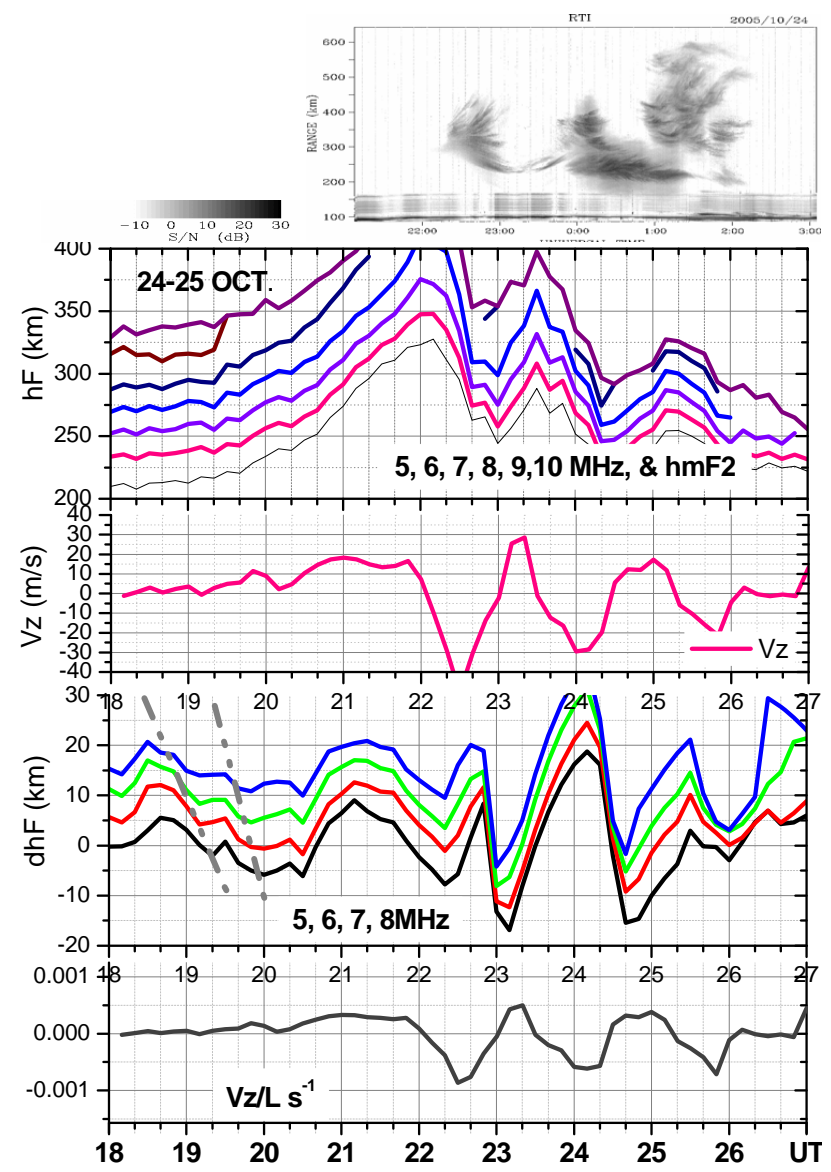

Fig. 2b. Plots similar to those of Fig. 2a, but for the evening-night period of 24-25 October 2005.

sunset (21:00 UT) did not present any clear downward phase propagation characteristics, indicating thereby rather weak GW activity as precursor to spread F. The lack of downward phase progression could mean little or no GW activity or insufficient measurement cadence because the GW frequency was close to $\mathrm{N}$ (buoyancy frequency). In the latter case, the GW would be close to evanescent mode and could contribute significant vertical motions, but without the telltale phase descent (see Fritts et al., 2008). In comparison to this, a clear GW signature was present on the evening of 24 October as can be verified from panel 4 in Fig. $2 b$ that presents the height oscillations for 24-25 October. The PRE vertical drift on this evening attained a peak value of only $18 \mathrm{~m} / \mathrm{s}$ by $21: 00 \mathrm{UT}$ and remained weak until its reversal to downward just after 22:00 UT. The corresponding growth rate factor remained at $3 \times 10^{-4} \mathrm{~s}^{-1}$ or smaller from 20:50 until 21:50, then turning negative after 22:00 UT. The development of the spread $\mathrm{F}$ at 22:15 as seen in the RTI map appears to be compatible with this growth rate. However, we will be showing later using model calculations, that spread $\mathrm{F}$ irregularities would not have occurred on this evening if the precursor GW characteristics were the same as they were on the evening of 23 


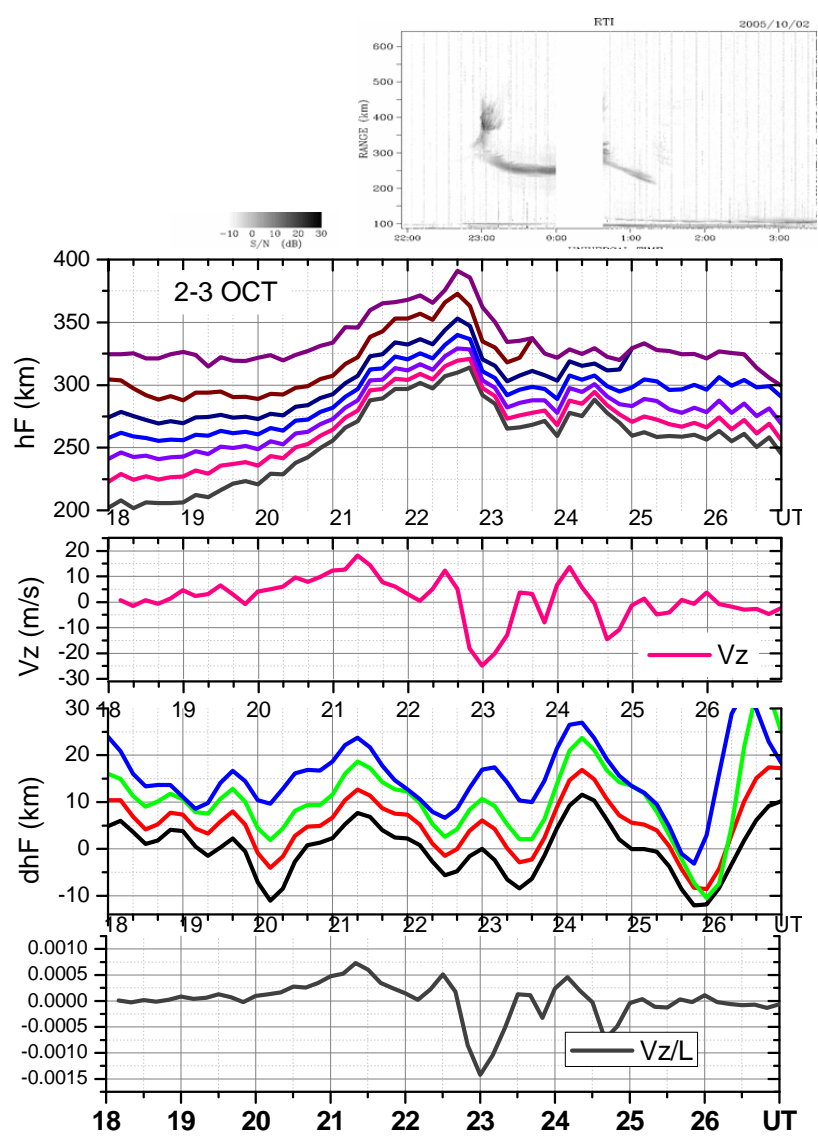

Fig. 3a. Plots similar to those of Fig. 2a and b, but for the eveningnight period of 2-3 October 2005.

October. In other words the irregularity development in this case was helped by the presence of GWs of significant amplitude seen on this evening.

Two other contrasting cases are presented in Fig. 3a, for the night of 2-3 October, and in Fig. $3 b$ for the night of 5-6 October. In the former case the irregularities were weak and of mostly bottom type, although beginning with a short lived penetration to $450 \mathrm{~km}$, whereas in the latter case the irregularities that began as "bottom type SpF" exhibited higher intensity including plume extension to the top side, as seen in the respective RTI maps. On the evening of 2 October, the vertical velocity reached a peak of $18 \mathrm{~m} / \mathrm{s}$ at 21:20 UT (18:20 LT) followed by a smooth decrease to zero by $22: 10 \mathrm{UT}(19: 10 \mathrm{LT})$. The growth rate factor attained a value of $7.5 \times 10^{-4} \mathrm{~s}^{-1}$ (corresponding to an irregularity growth time of $23 \mathrm{~min}$ ) which should have resulted in irregularity onset by about 21:45 UT if a significant precursor seed perturbation were to be present. But no spread F occurred for the following $90 \mathrm{~min}$. Irregularities with an altitude penetration up to $450 \mathrm{~km}$ (but short lived) developed after $90 \mathrm{~min}$, at 22:50 UT which appear to have been initiated/ caused by a second increase in $V z$, with growth rate peaking at 22:30 UT

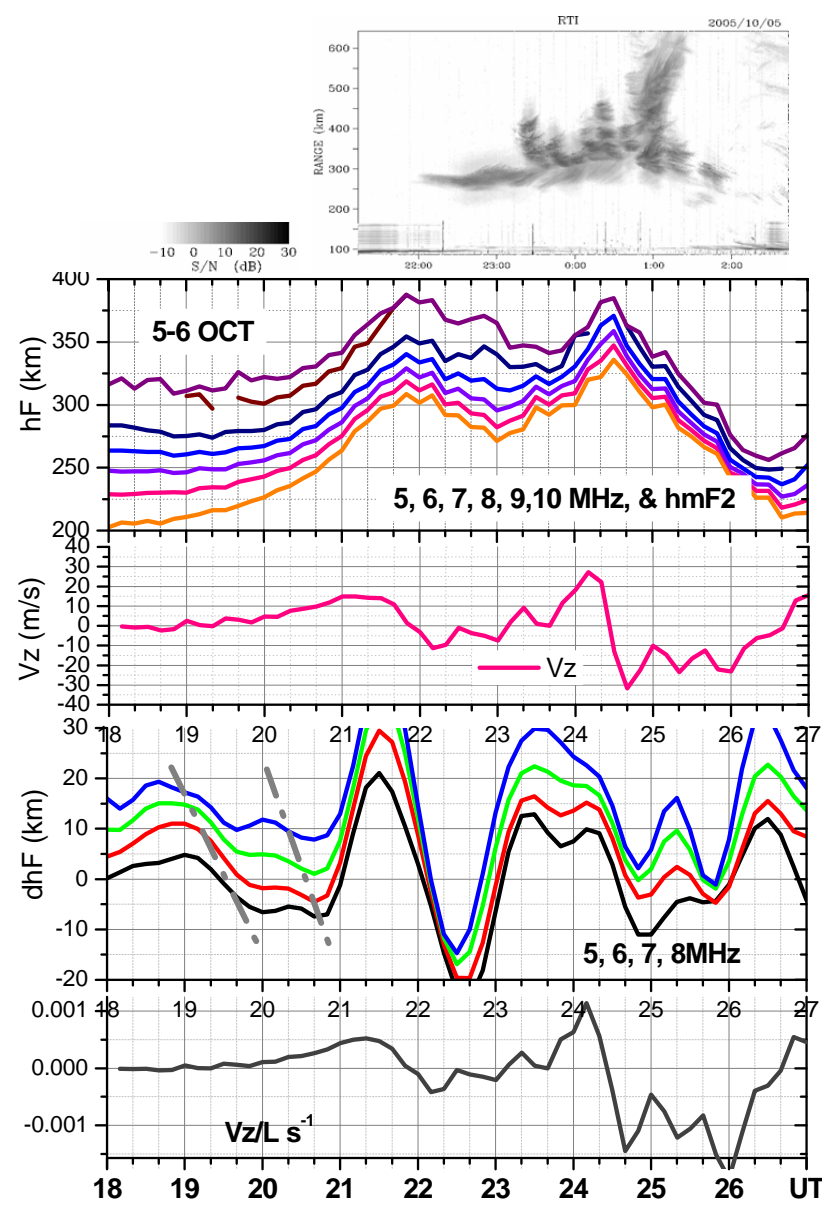

Fig. 3b. Plots similar to those of Fig. 2a and b, but for the eveningnight period of 5-6 October 2005.

(bottom panel of Fig. 3a). The rest of the night was mostly uneventful. The important point to note in this sequence is that a vertical drift peak at 21:20 UT that indicated an instability growth time of $23 \mathrm{~min}$ did not produce any irregularity development for about $90 \mathrm{~min}$ after the drift peak, and a second increase of the vertical drift at 22:30 appears to have contributed to the short lived radar plume at 22:50 UT. The precursor height fluctuations on this evening do not appear to indicate the presence of any significant GWs as the downward phase propagation in them is marginal. (Again, as mentioned before this by itself does not indicate an absence of GWs, but only that if present, they had an unresolved frequency or a very large vertical structure suggestive of evanescence.). We therefore believe that the absence of a clearly identifiable GW by the digisonde appears to be responsible for the non occurrence of an irregularity event in the bottom-side due to pre-reversal peak even though the linear growth rate did predict such occurrence. In contrast to this we note the clear presence of significant precursor GWs on the evening of 5 October (Fig. 3b). The vertical drift peak on this evening occurred also at 21:20 UT (as on 2 October) but with a smaller 
value of $14 \mathrm{~m} / \mathrm{s}$ only. The corresponding linear growth rate $\left(5 \times 10^{-4} \mathrm{~s}^{-1}\right)$ that was smaller as well, predicted irregularity development to start after 33 min which is indeed seen in the onset of the 5-m irregularity structure in the RTI map that was limited to the bottomside till 23:20 UT. We have strong reason to believe that the GW presence on this evening played a role in the bottom-side irregularity development that followed the pre-reversal drift enhancement and in subsequent bubble developments, in contrast to the evening of 2 October when in spite of a large growth rate condition, bottomside SF and bubble did not develop during the expected time for their developments (for about $90 \mathrm{~min}$ ) and were weak as well when they did occur at later times, under the condition of marginal gravity wave presence. The $5-\mathrm{m}$ structure soon erupted into topside plume by 23:20 UT probably aided by an increase in the $V_{y} / l_{0}$ growth rate factor as well as the large amplitude GWs (panels 4 and 5, Fig. 3b) that immediately preceded this growth. Thus from a comparison of the causeeffect sequences on these two days (just explained above), and further considering the nearly identical precursor behavior of the $h_{F}$ on these two days, we are tempted to conclude that the presence of GWs was a unique (and readily identifiable) factor that appears to have contributed to the rather prompt development of irregularities on the evening of 5 October in contrast to the evening of 2 October when a larger growth rate did not lead to irregularity development (or produced only weak spread F) in the absence of any clear precursor GWs.

\section{Discussion}

The SpreadFEx campaign was conducted during equinoctial months of low solar flux epoch (F10.7 in the range 75-80) and as such the evening thermospheric winds that control the ambient conditions for spread $\mathrm{F}$ occurrence (such as the prereversal enhancement in zonal electric field) must be dominated by the zonal components of these winds, which means that any possible influences in the ESF day-to-day variability that could arise from meridional/trans-equatorial winds (Abdu et al., 2006, 2009) through their modification of the flux tube integrated conductivities (Maruyama, 1988) can be ignored as is done here. What we analyze here are examples of spread F development, and its day-to-day variability, under precursor conditions representative of competing influences from varying combinations of the driving forces from prereversal vertical drift and GW features. The rate at which the polarization electric field builds up in a growing instability under the actions of the GWs and the PRE can be used to assess the expected intensity of an ESF event as being more or less likely to be influenced by one or the other of these driving forces.

In Appendix A, governing equations for a zonal polarization field $\delta E_{x}$ excited by RTI is derived using hydromagnetic equations, detailed derivation is presented in a recent submis- sion (Kherani et al, 2009b). An equation for $\delta E_{x}$ is obtained in the following form:

$\frac{\partial \delta E_{x}}{\partial t}-\gamma_{R} \delta E_{x}=s_{x}$

Where,

$\gamma_{R}=\left(-\frac{E_{o x}}{B_{o}}-W_{y}+\frac{g}{v_{i}}\right) \frac{1}{l_{o}} ;$

$s_{x} \approx \frac{B_{0} U_{0 x}}{l_{0}}\left(\frac{\delta W_{y}}{\kappa_{i}}-\delta W_{x}\right) ; 1 / l_{o}=d \log n_{o} / d y$

$U_{0 x}$ is the background zonal wind; $\delta W_{x, y}$, the zonal/ vertical perturbation winds;

$E_{0 x}$, the zonal electric field (PRE); $g, v_{i}, n_{o}$, the gravitational acceleration, ion-neutral collision frequencey and background electron density respectively; see Appendix A for more details.

\subsection{Growth rate evaluation and comparison with observation}

Equation (1) is the governing equation for the zonal component of the polarization field perturbation which is the principal field in the excitation of Rayleigh-Taylor instability. In the linear limit, it can be solved analytically and it has the following solution:

$\delta E_{x}=s_{x} / \gamma_{R}\left(\exp \gamma_{R} t-1\right)$

Here $\gamma_{R}$ is recognized as a growth rate of RTI and chosen to be constant at its peak value in the F region. The free energy in the ionosphere is in the form of current which flows mainly in zonal direction. To extract this energy, some kind of pumping source in this direction is desired. The term $s_{x}$ in Eq. (1) acts like a pumping source necessary to extract the free energy available in the ionosphere. It is in the form of wind perturbation $\delta W$ associated with a seeding gravity wave with propagation in zonal-vertical plane at the equator perpendicular to Earth's magnetic field. For $\gamma_{R} t \ll 1$ the source function, $s_{x}$ decides the initial amplitude of $\delta E_{x}$ which will subsequently grow or damp depending upon the nature of the growth rate $\gamma_{R}$. In the limit $\gamma_{R} t \ll 1, \delta E_{x}$ grows linearly with time and its evolution is mainly dictated by $s_{x}$. As $\gamma_{R} t \rightarrow 1, \delta E_{x}$ begins to increase exponentially which is the linear phase of the instability growth. It is noted that similar to $\gamma_{R}$, the source function $s_{x}$ is proportional to the vertical density gradient. It means that pumping source associated with GW is likely to maximize in the altitude region where growth rate of RTI maximizes. On this basis, GW induced perturbation may be regarded as an efficient seeding mechanism for RTI.

In present study, GW of tropospheric origin is considered. To infer the nature of GW in present study, we employ a $\mathrm{GW}$ vertical propagation model that describes the evolution with altitude of a GW with specified characteristics at tropospheric altitude (Kherani et al., 2009b). The model provides 

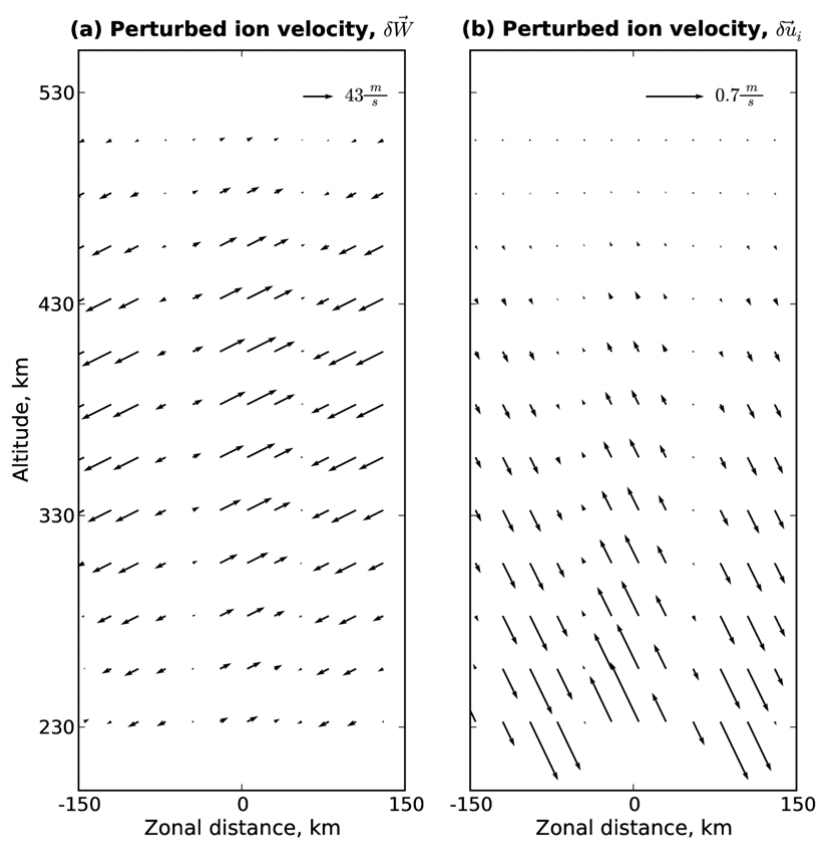

Fig. 4. (a) The distribution of amplitude $\delta W$ of GW; (b) and the perturbed ion velocity $\delta u_{i}$ in the $\mathrm{F}$ region at $t=0$.

vertical velocities associated with a $\mathrm{GW}$ at altitudes from 10 to $600 \mathrm{~km}$ with a given initial velocity fluctuation at the lower boundary. The GW associated horizontal velocities are obtained from the continuity equation (Eq. 10 of Fritts et al., 2008b). In the present study, the initial GW vertical velocity amplitude is assumed to be $0.5 \mathrm{~cm} / \mathrm{s}$ at $10 \mathrm{~km}$ height corresponding to buoyancy velocity of convective plumes. The vertical and horizontal wavelengths $\left(\lambda_{y, x}\right)$ are chosen to be two and four times larger than the scale height at each altitude. Given these wavelengths, the frequency $(\omega)$ of the GW is obtained from the dispersion relation $\omega^{2} \lambda_{x}^{2}=\left(N^{2}-\omega^{2}\right) \lambda_{y}^{2}$. This is one among few options by which the GW's parameters can be selected. An alternative approach is to fix the $\lambda_{y}$ and $\omega$ and calculate the $\lambda_{x}$ from above dispersion relation as was done in Sect. 3.1. The wave frequency estimated using the above approach is found in the range 10-60 min depending on the altitude. The wave resides in equatorial plane (x-y) perpendicular to magnetic field and moves obliquely at an angle $84^{\circ}$ from horizontal. The fluctuating wind $\delta \boldsymbol{W}$ associated with the GW is shown in Fig. 4a. It can be seen that $\delta \boldsymbol{W}$ maximizes in the $\mathrm{F}$ region and it is dominantly horizontal having horizontal wavelength in the range of $200-300 \mathrm{~km}$. The maximum horizontal and vertical winds associated with GW are of order of $40 \mathrm{~m} / \mathrm{s}$ and $10 \mathrm{~m} / \mathrm{s}$, respectively. This $\mathrm{GW}$ introduces a perturbation $\delta \boldsymbol{U}$ in the ion velocity (given in appendix) and it is plotted in Fig. 4b. It is noted that $\delta U$ is dominantly vertical having a magnitude of $0.5-1 \mathrm{~m} / \mathrm{s}$ in the bottomside F-region.

To infer the relative importance of $s_{x}$ and $\gamma_{R}$, we study the evolution of $\delta E_{x}$ based on input parameters obtained from the observations. We will consider the results on the two selected pairs of days, 23-24 October 2005 of Fig. 2a and b and 2-5 October 2005 of Fig. 3a and b. Each pair consists of days when the prereversal enhancement, $F$ layer bottom side gradients and wave signatures, are very different just prior to spread F. (While the F layer heights were different on former pair of days they were rather close by on the latter pair of days). On the evening of 23 October the $V_{y} / l_{o}$ factor and the F layer heights were larger than on the evening of 24 October. On the other hand, the GW signatures were more prominent in the latter than in the former case. Spread F was observed on both evenings, but it was more extended in height (as topside plumes) and stronger on 23 October and less extended in height and weaker on 24 October. In order to evaluate the role of $\mathrm{GW}$ in the contrasting features on this day pair, we calculated the evolution of the perturbation electric field in the instability development for the following three cases of the input parameters:

Case 1a: Ambient ionospheric conditions corresponding to 23 October and GW amplitude $\delta W=\delta W_{o}$;

Case 1b: Ambient ionospheric conditions corresponding to 24 October and GW amplitude $\delta W=\delta W_{o}$;

Case 1c: Ambient ionospheric conditions corresponding to 24 October and GW amplitude $\delta W=2.5 \delta W_{o}$;

(An amplitude of GW on each night is derived using observed time variation of bottom-side F layer height $\left(h_{F}\right)$. The time rate of change of $h_{F}$ at given time represents perturbed upward ion velocity $\delta u_{y}$. For one hour periodic modulation observed during 19:00-21:00 UT, this time rate is estimated on each night and corresponding wind perturbation $\delta w_{x} \approx \frac{\Omega}{v_{i}} \delta u_{y}$ is obtained. It is found that the estimated $\delta u_{y}$ is of order of $0.8 \mathrm{~m} / \mathrm{s}$ and $2 \mathrm{~m} / \mathrm{s}$ on 23 and 24 October, respectively. On this basis, $\delta W$ is chosen to be $\delta W_{o}$ and $2.5 \delta W_{o}$ on 23 and 24 October, respectively, where $\delta W_{o}$ and corresponding perturbed ion velocity $\delta u$ are shown in Fig. $4 \mathrm{a}-\mathrm{b}$.)

The value of $\delta W_{0}$ used in the model calculation was the same for both the day pairs analyzed here, and it corresponds to a wind perturbation that produced ion perturbation velocity that was marginally detectable by the digisonde in the plots of Figs. 2a and 3a. As compared to this the GW oscillations in the plots of Figs. $2 \mathrm{~b}$ and $3 \mathrm{~b}$ presented measurable amplitudes and a dominant period of the order of one hour. In the present model calculations we have used the value for $\delta W_{0}$ as obtained in Fig. $4 \mathrm{a}$ which is of the order of $40 \mathrm{~m} / \mathrm{s}$ in the F layer bottomside, near $320 \mathrm{~km}$ at which the seeding altitude starts. It may be noted that the corresponding ion perturbation velocity as sown in Fig. $4 \mathrm{~b}$ is $\sim 0.8 \mathrm{~m} / \mathrm{s}$ which is compatible with an approximate estimate of such velocity obtained from a one-hour smoothed $h_{F}$ oscillation features that existed just prior to sunset hours in Figs. 2a and 3a.

From the observation we obtain the following three parameters:

$R_{v_{y} / l_{o}}=\left(v_{y} / l_{o}\right)_{23} /\left(v_{y} / l_{o}\right)_{24} \approx 2 ;$

$R_{h}=(h)_{23} /(h)_{24} \approx 1.2$; 
$R_{\delta W}=(\delta W)_{23} /(\delta W)_{24} \approx 2.5$

Here $R_{v_{y} / l_{o}}, R_{h}$ and $R_{w}$ represent the ratios of $V_{y} / l_{o}$, the bottom-layer height and the amplitude of GWs, respectively, between 23 and 24 October.

In Fig. 5 (top panel) the growth of $\delta E_{x}$ is plotted for Cases 1a-1c. We may note that the values of $\delta E_{x}$ for the linear phase of the instability growth shown here are smaller than in actual instability development wherein a nonlinear phase may soon set in for vertical growth into a topside bubble. The nonlinear process of the instability growth is discussed in a companion paper by Kherani et al. (2009a). For Case 1a (conditions of 23 October) in Fig. 5a, $\delta E_{x}$ grows exponentially within $1000 \mathrm{~s}$ (solid curve), thereby indicating/predicting the subsequent favorable condition for development of a bubble, as is indeed observed (Fig. 2a). It is to be noted in this case, however, that the presence of GWs is not clear in the digisonde data and therefore the amplitude of the GW wind oscillation is taken as $\delta W_{0}$ in the calculation of $\delta E_{x}$. For Case $1 \mathrm{~b}$ that used the input parameters for 24 October in combination with the GW characteristics of 23 October $\left(\delta W_{0}\right)$, the instability evolution does not reach to exponential phase (dash curve) indicating that conditions were unfavorable for the subsequent bubble, or for bottom-side irregularity, development. For Case 1c, that used the input parameters for 24 October in combination with the GW parameters also for the same day $\left(\delta W=2.5 \delta W_{0}\right)$, the evolution again acquires the exponential growth within $1000 \mathrm{~s}$ (solid line with "+" curve) though a bit slower than in Case 1a. This slower evolution of $\delta E_{x}$ appears to be consistent with the post sunset radar plume development that was restricted in altitude mainly to the bottom-side. The contrasting behavior of the exponential growth in Case 1c and the non-exponential growth in Case $1 \mathrm{~b}$ clearly demonstrates the importance of GW amplitudes (through $s_{x}$ ) in the growth of RTI, in a manner that is consistent with the observational results from radar and digisonde.

To examine the influence of GW on the day pair, 2 and 5 October (Fig. 3a and b), the following cases are considered:

Case 2a: Ambient ionospheric conditions corresponding to 2 October and $\mathrm{GW}$ amplitude $\delta W=\delta W_{o}$;

Case 2b: Ambient ionospheric conditions corresponding to 5 October and $\mathrm{GW}$ amplitude $\delta W=\delta W_{o}$;

Case 2c: Ambient ionospheric conditions corresponding to 5 October and $\mathrm{GW}$ amplitude $\delta W=4 \delta W_{o}$;

In Fig. 5 (bottom panel) the growth of $\delta E_{x}$ is plotted for Cases $2 \mathrm{a}, 2 \mathrm{~b}$ and $2 \mathrm{c}$. We note that it grows exponentially for all three cases but faster for Case $2 a$ and slowest for Case $2 b$. For Case $2 \mathrm{c}, \delta E_{x}$ attains intermediate amplitude and shows a tendency of rapid growth in spite of less-favorable ambient conditions. (in terms of $V_{y} / l_{o}$ ). This aspect resulted from use of larger $\mathrm{GW}$ amplitude (in the calculation) for Case $2 c$ that was present on this evening. Thus we verify here as we did from the first pair of days that the presence of GWs does indeed lead to enhanced irregularity development
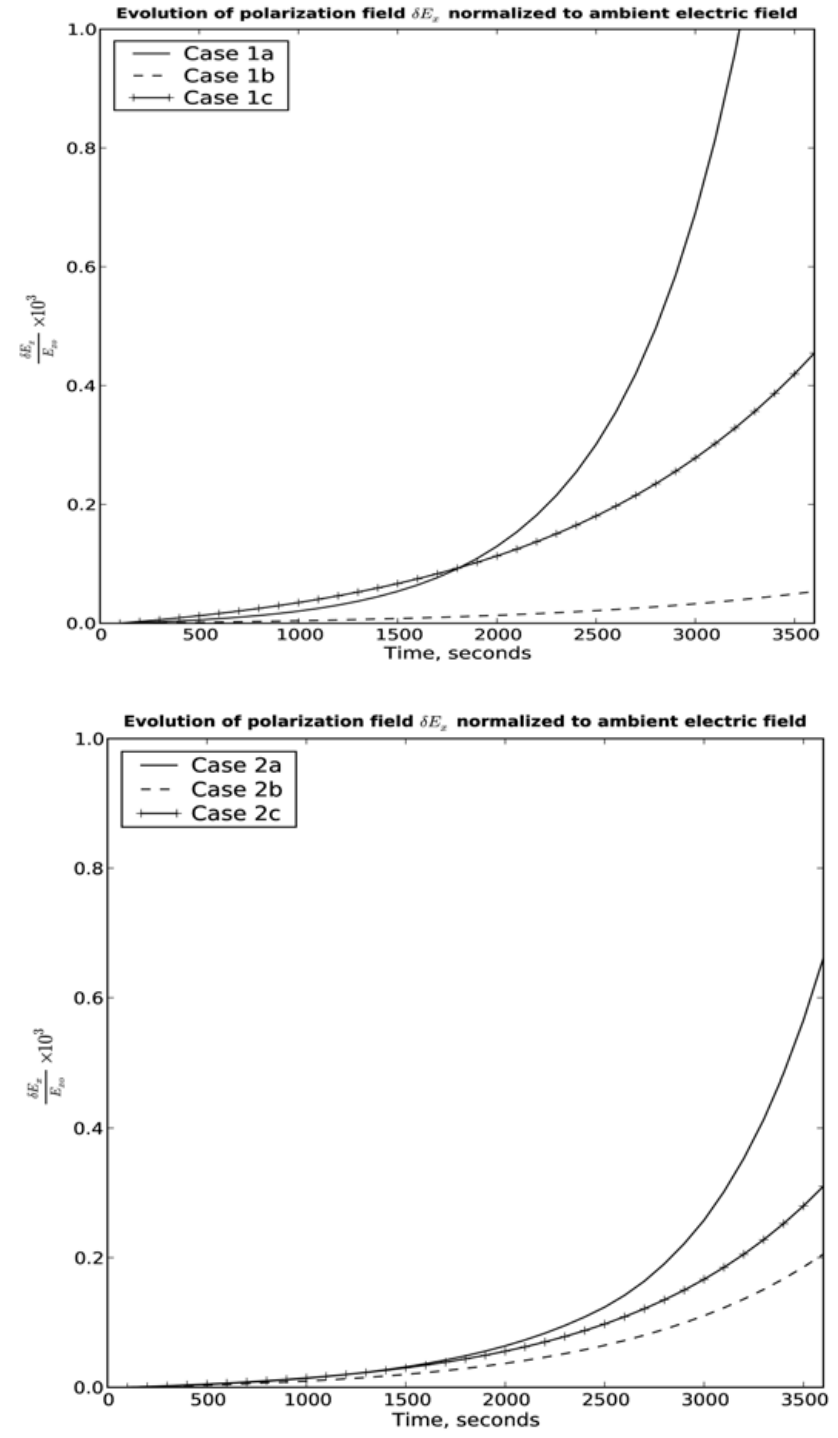

Fig. 5. Top panel: the time evolution of the zonal component of the polarization electric field, $\delta E_{x}$, controlled by $\gamma_{R}$ and gravity wave winds for the three cases discussed in the text as follows: the solid curve represents the result corresponding to the input parameters for the evening of 23 October in which the GW strength is $\delta W_{0}$ (Case 1a); the dash curve shows the results from input parameters for 24 October except that the GW characteristics are those of 23 October (Case 1b); and the line with "+" curve is the result when the gravity wave contribution in the Case $1 \mathrm{~b}$ curve was increased to correspond to that observed on 24 October, with $\delta W=3 \delta W_{0}$ (Case 1c). Bottom panel: the results similar to that of the top panel but for the day pair 2 and 5 October. The solid curve represents the result corresponding to the input parameters for the evening of 2 October in which the GW strength is $\delta W_{0}$ (Case 2a); the dash curve shows the results from input parameters for 5 October except that the GW characteristics are those of 2 October (Case 2b); the line with "+" curve is the result when the gravity wave contribution in the Case $2 b$ curve was increased to correspond to that observed on 5 October, with $\delta W=4 \delta W_{0}$ (Case 1c). 
as predicted from model calculations and in agreement with the observations.

A further inter-comparison of the results between the two pairs of days brings out some important aspects of the relative/competing roles of the gravity waves, the gravity and the $F$ layer height and vertical drift in the contrasting conditions of instability development in these cases. For example, in the case of 23 October (Fig. 2a) and 2 October (Fig. 3a) the same gravity wave amplitude $\left(\delta W_{0}\right)$ was used to calculate the $\delta E_{x}$ time evolution. The peak growth rate factor $\left(V_{y} / l_{o}\right)$ in the former case was smaller $\left(\sim 5 \times 10^{-4} \mathrm{~s}^{-1}\right)$ than in the latter case $\left(\sim 7 \times 10^{-4} \mathrm{~s}^{-1}\right)$. Yet topside penetrating bubble developed within $\sim 35$ min after the peak growth rate in the former case with no instability developing till $\sim 90 \mathrm{~min}$ after the peak growth rate in the latter case (in which as mentioned before, the short lived and weak bubble that developed at 23:00 UT/20:00 LT was caused by a second growth rate increase that peaked at 22:30 UT just preceding this event). This clearly shows that the gravity term, $g / v_{i} l_{o}$, enhanced by the larger $\mathrm{F}$ layer heights in the evening, contributed to the rather rapid exponential growth in $\delta E_{x}$ (Fig. 5 Case 1a) and hence to the prompt bubble development on the evening of 23 October.

We now compare the results of 2 October for which GW amplitude was $\delta W_{0}$ with that of 5 October for which this amplitude was as large as $4 \delta W_{0}$. On 2 October the exponential growth of the polarization electric field was less pronounced (than on 23 October) and as a result bubble did not develop (till $\sim 90 \mathrm{~min}$ ). On 5 October the $\delta E$ exponential growth, though enhanced by the GW amplitude of $4 \delta W 0$, was still smaller than on 2 October, but instability did develop (within $\sim 35 \mathrm{~min}$ ) in the form of bottom type spread F. The role of gravity waves in this instability development appears to be evident from the following reasoning. The polarization electric field, in its early stage of the growth can be represented by a simplified form of the Eq. (2) for $t \ll 1 / \gamma_{R}$ as given by:

\section{$\delta E_{x}=s_{x} t$}

The $s_{x}$ contains contributions from both the GW perturbation winds and the background zonal winds that determine the intensity of the $\delta E_{x}$ in its early stage of develpment (as also noted before). On 5 October, $s_{x}$ was four times larger than 2 October and thus bottomside irregularties developed on 5 October inspite of the fact that the growth rate is slower than 2 October. From a careful examination of the radar map on 5 October, in Fig. 3a we make a significant observation that the bottom type spread $\mathrm{F}$ local time pattern immediately starting from its initiation at $\sim 22: 00$ UT (19:00 LT) contains a wave structure with period of the order 1-h that appears to be compatible with the precursor GW structure detected in the digisonde data. Thus the role of GW winds appears to be manifesting itself in the structring of the bottom type spread $\mathrm{F}$ in this case. Its development into topside penetrating bubbles did not evolve due to the inadequate growth rate factor
$\gamma_{R}$ that remained low till $\sim$ 23:00 UT. (An ensuing rapid increaae in its value produced the bubble event at 23:20 UT which is discussed in detail by Kherani et al., 2009). We should point out here that the zonal background wind which is eastward at this time could have an intensity of the order of $70 \mathrm{~m} / \mathrm{s}$ (Abdu et al., 2005) corresponding to the prereversal drift of $\sim 15 \mathrm{~m} / \mathrm{s}$ registered on this evening. Such a wind, though of relatively smaller intensity, may also have contributed to the bottom type spread F development on this evening according to the wind driven instabilty mechanism discussed by Kudeki et al. (2007).

Linear analysis presented above only ensures initial growth of CII without taking into account temporal variations of ambient parameters such as vertical drift and rapidly varying density owing to the instability itself. These variations, depending upon its nature, may or may not allow exponential growth to continue. This aspect can be examined only with a nonlinear simulation which is presented in companion paper (Kherani et al., 2009a). The results of the nonlinear simulation confirm $\delta E_{x}$ growth characteristics presented above except for Case $2 b$. For this case, simulation shows suppression of instability caused by rapid downward motion of ionosphere after pre-reversal peak (Fig. 3b). It is also found that instability keeps growing and gives rise to bubble for Case $2 \mathrm{c}$ where elevated GW amplitude overcomes damping effects of rapid downward motion, and contributes to recurring increases in the vertical drift, on 5 October 2005. The first such bubble development can be seen at $\sim 23: 20$ UT (20:20 LT) on this evening.

We may point out that the GW characteristics (wavelength, period and wind velocities, etc.) used in the model calculations may not be exactly the same as those that must have been present on the days of these observation. This is because we do not have measurements of the GW wind fields over this or any other longitude sector of the equatorial region for the precursor conditions on which to base the values used in our calculation. We expect that this situation does not impact much on the important findings from this study because (a) the gravity wave parameters inferred from the present observational data and that adopted in the model calculations are in general consistent with the theoretical predictions as presented in the companion paper by Fritts et al. (2008) and the results by Kherani et al. (2009b) and (b) they are based on comparing the results for selected pairs of days. In any case the agreement between our findings as inferred from the observations and that obtained from the model calculations tend to reinforce such an expectation. We need, however, to pursue efforts for more quantitative evaluations on a minimum level of GW intensity required to seed an instability growth for a specific prereversal enhancement intensity and vice versa. This is a challenging task since the lack of full definition in terms of perturbation wavelengths, periods, and the zonal and vertical winds of the GWs based on observational data makes it difficult to assess the question of a minimum level of geophysical noise/vis-a-vis gravity wave amplitudes. 
In the line of experimental/observational approach used in this paper, further perfection towards a better quantification of the measurable parameters can be achieved in different ways. For example, the operational mode of the digisonde can be set up to yield better resolutions in height and density of the bottomside $\mathrm{F}$ layer. Correlation/association between GW oscillation detected by the digisonde at Fortaleza with the spread F diagnosed over Sao Luis, (separated in longitude by $650 \mathrm{~km}$ ), can be improved by operating additional one or two equipments at strategically spaced smaller distances from the one at a dip equatorial site. A still broader approach would call for a coordinated multi-instrument campaign efforts to collect diverse data sets including the thermospheric zonal, meridional and vertical wind fields of the bottomside $\mathrm{F}$ region with GW resolution, covering the precursor period into the post sunset hours together with the high resolution diagnostics on background ionospheric conditions and the irregularity structuring. It is of particular interest to evaluate and resolve the question of the possibly competing contributions to the instability generation arising from eastward zonal wind, through the PRE and the wind driven instability as discussed by Kudeki et al. (2007), and that arising from perturbation winds due to GWs in combination with the PRE in the line discussed in this paper. Radars, Digisondes, Fabri-Perrot interferometers and optical imagers etc. are to be deployed at strategic locations extending from dip equator to low latitudes as well as covering longitudes considering the dominant zonal propagation of the GWs. Such measurements could be complemented with observation by satellites of opportunity such as for example the C/NOFS which is now in orbit.

\section{Conclusions}

We have analyzed radar and digisonde data collected during the SpreadFEx campaign to investigate the importance of GW influences in initiating equatorial spread $\mathrm{F}$ irregularity development during post dusk hours. The analysis focused on the ambient conditions of the evening IT, in terms of the prereversal enhancement in zonal electric field/vertical drift, the F layer bottom-side density gradients, and the density fluctuations in the form of GWs that were present, as precursors to post sunset spread $\mathrm{F}$ development. The observational findings on the possible $\mathrm{GW}$ influence on spread $\mathrm{F}$ instability initiation were modeled using the theory for the linear growth of the RTI process, with encouraging results. The main conclusions from this study are the following:

1. The features of GWs as observed in their signatures in the F layer bottom-side densities are in general agreement with those predicted by theory for GWs propagating from tropospheric convective sources as identified from complementary experiments conducted during the SpreadFEx campaign;
2. A large degree of day-to-day variability was present in the precursor GW characteristics as seen in their signatures in $\mathrm{F}$ layer densities as well as in the prereversal vertical drift;

3. GW winds can contribute significantly to the polarization electric fields controlling the instability growth, depending on the $\mathrm{F}$ layer vertical drift velocity, the layer height and height gradient in density at the $\mathrm{F}$ layer bottom-side;

4. Spread F instability initiation and continuing development depend upon the precursor condition of the evening prereversal electric field/vertical drift, bottomside density gradients and a density perturbation as a seed source, that complement/compete one another. It is found from the cases analyzed here that radar 5-m irregularity plumes, indicative of the presence of topside bubbles, can be generated for precursor vertical drift velocities exceeding $30 \mathrm{~m} / \mathrm{s}$ even when the precursor GWs induced density oscillations are marginally detectable by the digisonde;

5. When the instability growth rate due to the control factors arising from the evening vertical drift $(V z / L)$ and gravity $\left(g / v_{i n} L\right)$ is insufficient, or marginal, for spread $\mathrm{F}$ development, the presence of GW winds can enhance the growth of instability polarization electric fields leading to spread F irregularity development either as bottom type spread F or vertically penetrating plumes/density depletions. The agreement between the theory based model calculation and the observational data presented here provides evidence that GW induced seed perturbation do indeed operate in the process of spread $\mathrm{F}$ initiation and the ensuing evolution to form topside bubbles;

6. The results suggest that GW winds could be an important driver for bottom type spread F development whose evolution to topside bubbles is dependent on the growth rate factors determined also by the vertical drift and $\mathrm{F}$ layer heights (gravity term);

7. In view of the evaluation by Fritts et al. (2008) that GW characteristics at the thermospheric/ $\mathrm{F}$ layer heights are consistent with those of the GWs propagating upward from tropospheric convective regions, the present set of results appears to provide evidence that tropospheric GWs can be an important cause of the spread F day-today variability.

Further analysis is continuing towards establishing more quantitative relationship between the different control parameters related to spread $\mathrm{F}$ development processes. 


\section{Appendix A}

In order to derive the governing equation for perturbed electric field $\delta E$, following equation, derived using Maxell's equation under divergence-free current density condition, is employed: we use the wave form of the Maxwell's equation for perturbed electric field $\delta E$ :

$\nabla^{2} \delta E-\frac{1}{c^{2}} \frac{\partial^{2} \delta E}{\partial t^{2}}-\nabla(\nabla . \delta E)-\mu_{o} \frac{\partial \delta J}{\partial t}=0$

where only first order terms are kept. The divergence-free current density condition $\nabla \cdot \delta J=0$ implies $\nabla \cdot \delta E=0$ (Kherani et al., 2009c). The unstable GRTI wave is low-frequency wave (Kelley, 1989) for which the second term in the above equation can be ignored. The above equation thus reduces to following form:

$\nabla^{2} \delta E-\mu_{o} \partial \delta J / \partial t=0$

Here $\delta J$ represents the total perturbed current driven by electrical and mechanical forces and is given by the following expression:

$\delta J=\sigma^{o} . \delta E+\delta \sigma \cdot E_{o}+\delta j_{m}$

where $\sigma=\sigma^{0}+\delta \sigma$ is the conductivity tensor and $\delta j_{m}$ is the current density caused by the mechanical and gravitational forces. In the linearised form, this can be written as follows:

$\delta j_{m}=e n_{o}\left(\delta u^{i}-\delta u^{e}\right)+e\left(u_{o}^{i}-u_{o}^{e}\right) \delta n=e n_{o} \Delta \delta u^{M}+e \Delta u_{o}^{M} \delta n$

The governing Eq. (A2) is derived in Cartesian plane (x (west)-y (up)) perpendicular to the magnetic field $B_{o} \hat{z}$. Substituting $\delta J$ in Eq. (2) and neglecting ion/electron inertia lead to following equation for $\delta E$

$$
\begin{aligned}
& -\frac{\partial \delta E_{x}}{\partial t}-\frac{E_{o x}}{\sigma_{P}^{o}} \frac{\partial \delta \sigma_{P}}{\partial t}-\frac{e}{\sigma_{P}^{o}} \Delta u_{o x}^{M} \frac{\partial \delta n}{\partial t}=0 \\
& -\frac{\partial \delta E_{y}}{\partial t}-\frac{E_{y}^{o}}{\sigma_{P}^{o}} \frac{\partial \delta \sigma_{P}}{\partial t}-\frac{e}{\sigma_{P}^{o}} \Delta u_{o y}^{M} \frac{\partial \delta n}{\partial t}=0
\end{aligned}
$$

Provided, following conditions are satisfied:

$$
\begin{aligned}
& \frac{1}{\mu_{o} \sigma_{p}} \nabla^{2} \delta E_{x}-\frac{\sigma_{H}^{o}}{\sigma_{p}^{o}} \frac{\partial \delta E_{y}}{\partial t}-\frac{1}{\sigma_{p}^{o}} \frac{\partial \sigma_{H}^{o}}{\partial t} \delta E_{y}=0 ; \\
& \frac{1}{\mu_{o} \sigma_{p}} \nabla^{2} \delta E_{y}+\frac{\sigma_{H}^{o}}{\sigma_{p}^{o}} \frac{\partial \delta E_{x}}{\partial t}+\frac{1}{\sigma_{p}^{o}} \frac{\partial \sigma_{H}^{o}}{\partial t} \delta E_{x}=0
\end{aligned}
$$

The conditions (A4-A5) lead to the dispersion relation for the shear Alfven waves (by assuming $\delta=\delta \exp [i(k . r-\omega t)]$ ) which is discussed by Basu (2004) in the context of GRTI. We now focus on the governing equation for zonal perturbed field $\delta E_{x}$ in Eq. (A2) which is the principal field associated with GRTI.

$$
\frac{\partial \delta E_{x}}{\partial t}+\frac{e}{\sigma_{P}}\left(\Delta U_{o x}^{M}+\Delta U_{o x}^{E}\right) \frac{\partial \delta n}{\partial t}=0
$$

Here, $\Delta u_{o}^{E}$ and $\Delta u_{o}^{M}$ are the relative motion of ion-electron caused by electrical and mechanical forces respectively.

$$
\frac{e}{\sigma_{P}^{o}} \Delta u_{o x}^{E}=\frac{e}{\sigma_{P}^{o}} \frac{\sigma_{P}^{o} E_{o x}+\sigma_{H}^{o} E_{o y}}{e n_{o}} \approx \frac{1}{n_{o}} E_{o x}
$$

and $\frac{e}{\sigma_{P}^{o}} \Delta u_{o x}^{M}=\frac{e}{\sigma_{P}^{o} \kappa_{i}}\left(W_{y}-\frac{g}{v_{i n}}\right) \approx \frac{B_{0}}{n_{0}}\left(W_{y}-\frac{g}{v_{i n}}\right) ; \sigma_{P}^{o}=\frac{n_{o} e}{B_{o} \kappa_{i}}$

Here $W$ is the wind, $E_{0}$ is the ambient electric field in the ionosphere. The time-derivative of perturbed density $\delta n$ in (A6) can be replaced by the spatial-derivative of perturbed particle flux using ion continuity equation:

$$
\frac{\partial \delta n}{\partial t}=-\delta U_{y} \frac{\partial n_{o}}{\partial y}
$$

where

$$
\begin{aligned}
\delta U_{y} & =\frac{1}{1+\kappa_{i}^{2}}\left(-\kappa_{i}^{2} \frac{\delta E_{x}}{B_{0}}+\kappa_{i} \frac{\delta E_{y}}{B_{0}}-\kappa_{i} \delta W_{x}+\delta W_{y}\right) \\
& \approx-\frac{\delta E_{x}}{B_{o}}+\frac{1}{\kappa_{i}}\left(\frac{\delta W_{y}}{\kappa_{i}}-\delta W_{x}\right)
\end{aligned}
$$

The governing equation for $\delta E_{x}$ can finally be written as follows:

$$
\frac{\partial \delta E_{x}}{\partial t}-\gamma_{R} \delta E_{x}=s_{x}
$$

where $\quad \gamma_{R}=\left(-\frac{E_{o x}}{B_{o}}-W_{y}+\frac{g}{v_{i}}\right) \quad \frac{d \log n_{o}}{d y} ; \quad s_{x}=\frac{e \Delta U_{o x}}{\sigma_{P}} \frac{d n_{o}}{d y} \frac{1}{\kappa_{i}}$ $\left(\frac{\delta W_{y}}{\kappa_{i}}-\delta W_{x}\right) \approx \frac{B_{0} U_{0 x}}{l_{0}}\left(\frac{\delta W_{y}}{\kappa_{i}}-\delta W_{x}\right)$.

Acknowledgements. The authors wish to acknowledge the supports from FAPESP through the project 1999/00437-0, and CNPq through grants no. 502804/2004-1, 500271/2003-8. The SpreadFEx field program and supporting data analyses and modeling were also supported by NASA contracts NNH04CC67C and NAS5-02036 and AFOSR contract FA9550-06-C-0129.

Topical Editor U.-P. Hoppe thanks two anonymous referees for their help in evaluating this paper.

\section{References}

Abdu, M. A.: Outstanding problems in the equatorial ionospherethermosphere system relevant to spread F, J. Atmos. Solar Terr. Phys., 63, 869-884, 2001.

Abdu, M. A., Batista, I. S., Kantor, I. J., and Sobral, J. H. A.: Gravity Wave Induced Ionization Layers in the Night F-Region at Low Latitudes, J. Atmos. Terr. Phys., 44, 759-767, 1982.

Abdu, M. A., Batista, I. S., Takahashi, H., MacDougall, J., Sobral, J. H., Medeiros, A. F., and Trivedi, N. B.: Magnetospheric disturbance induced equatorial plasma bubble development and dynamics: A case study in Brazilian sector, J. Geophys. Res., 108(A12), 1449, doi:10.1029/2002JA009721, 2003.

Abdu, M. A., Medeiros, R. T., Bittencourt, J. A., and Batista, I. S.: Vertical ionization drift velocities and range spread $F$ in the evening equatorial ionosphere, J. Geophys. Res., 88, 399-402, 1983. 
Abdu, M. A., Iyer, K. N., de Medeiros, R. T., Batista, I. S., and Sobral, J. H. A.: Thermospheric meridional wind control of equatorial spread $\mathrm{F}$ and evening prereversal electric field. Geophys. Res. Lett., EUA, 33, L07106, 1-4, 2006 a.

Abdu, M. A., Batista, I. S., Reinisch, B. W., Sobral, J. H. A., and Carrasco, A. J.: Equatorial F region evening vertical drift, and peak height, during southern winter months: A comparison of observational data with the IRI descriptions, Adv. Space Res., 37, 1007-1017, 2006b.

Abdu, M. A., Batista, I. S., Reinisch, B. W., de Souza, J. R., Sobral, J. H. A., Bertoni, F., Pedersen, T. R., Medeiros, A. F., Schuch, N. J., de Paula, E. R., and Conjugate Point Equatorial Experiment (COPEX) Campaign in Brazil: Electrodynamics highlights on spread $\mathrm{F}$ development conditions and day-to-day variability, J. Geophys. Res., in press, 2009.

Alam Kherani, E., Abdu, M. A., de Paula, E. R., Fritts, D. C., Sobral, J. H. A., and de Meneses Jr., F. C.: The impact of gravity waves rising from convection in the lower atmosphere on the generation and nonlinear evolution of equatorial bubble, Ann. Geophys., 27, 1657-1668, 2009a,

http://www.ann-geophys.net/27/1657/2009/.

Batista, I. S., Abdu, M. A., and Bittencourt, J. A.: Equatorial Fregion vertical plasma drifts: Seasonal and longitudinal asymmetries in the American sector, J. Geophys. Res., 91, 12055-12064, 1986.

Bertoni, F., Batista, I. S., Abdu, M. A., Reinisch, B. W., and Kherani, E. A.: A comparison of ionospheric vertical drift velocities measured by digisonde and incoherent scatter radar at the magnetic equator, J. Atmos. Sol. Terr. Phys. 68(16), 1851-1852, 2006.

Farley, D. T., Balsley, B. B., and Woodman, R. F.: Equatorial spread F - Implications VHF radar observations, J. Geophys. Res., 75(34), 7199-7216, 1970.

Farley, D. T., Bonelli, E., Fejer, B. G., and Larsen, M. F.: The prereversal enhancement of the zonal electric field in the equatorial ionosphere, J. Geophys. Res., 91, 13723-13728, 1986.

Fejer, B. G., Scherliess, L., and de Paula, E. R.: Effects of the vertical plasma drift velocity on the generation and evolution of equatorial spread F, J. Geophys. Res., 104, 19859-19870, 1999.

Fejer, B. G., de Paula, E. R., Gonzalez, S. A., and Woodman, R. F.: Average vertical and zonal F-region plasma drifts over Jicamarca, J. Geophys. Res., 96, 13901-13906, 1991.

Fritts, D. C., Abdu, M. A., Batista, B. R., Batista, I. S., Batista, P. P., Buriti, R., Clemesha, B. R., Dautermann, T., de Paula, E. R., Fechine, B. J., Fejer, B. G., Gobbi, D., Haase, J., Kamalabadi, F., Kherani, E. A., Laughman, B., Lima, P. P., Liu, H.-L., Medeiros, A., Pautet, P.-D., Riggin, D. M., Rodrigues, F. S., São Sabbas, F., Sobral, J. H. A., Stamus, P., Takahashi, H., Taylor, M. J., Vadas, S. L., Vargas, F., and Wrasse, C. M.: Overview and summary of the Spread F Experiment (SpreadFEx), Ann. Geophys., 27, 2141-2155, 2009, http://www.ann-geophys.net/27/2141/2009/.

Fritts, D. C., Vadas, S. L., Riggin, D. M., Abdu, M. A., Batista, I. S., Takahashi, H., Medeiros, A., Kamalabadi, F., Liu, H.-L., Fejer, B. G., and Taylor, M. J.: Gravity wave and tidal influences on equatorial spread $\mathrm{F}$ based on observations during the Spread $\mathrm{F}$ Experiment (SpreadFEx), Ann. Geophys., 26, 3235-3252, 2008, http://www.ann-geophys.net/26/3235/2008/.

Fritts, D. C. and Vadas, S. L.: Gravity wave penetration into the thermosphere: sensitivity to solar cycle variations and mean winds, Ann. Geophys., 26, 3841-3861, 2008 http://www.ann-geophys.net/26/3841/2008/.

Hanson, W. B., Cragin, B. L., and Dennis, A.: The effect of vertical drift on the equatorial F - region stability, J. Atmos. Terr. Phys., 48, 205-212, 1986.

Heelis, R. A., Kendall, P. C., Moffet, R. J., Windle, D. W., and Rishbeth, H.: Electrical coupling of the $\mathrm{E}$ and $\mathrm{F}$ regions and its effect on the $\mathrm{F}$ region drifts and winds, Planet. Space Sci., 22, 743-756, 1974.

Hines, C. O.: Internal atmospheric gravity waves at ionospheric heights, Can. J. Phys., 38, 1441-1481, 1960.

Huang, C. S. and Kelly, M. C.: Nonlinear evolution of equatorial spread F 2. Gravity wave seeing of Rayleigh- Taylor instability, J. Geophys. Res., 101(A1), 293-302, 1996a.

Huang, C. S. and Kelly, M. C.: Nonlinear evolution of equatorial spread F 4. Gravity waves, velocity shear, and day-to-day variability, J. Geophys. Res., 101(A11), 24521-24532, 1996 b.

Huang, X. and Reinisch, B. W. Vertical electron density profiles from the digisonde networks, Adv. Space Res., 18(6), 121-129, 1996.

Hysell, D. L., Kelley, M. C., Swartz, W., and Woodman, R.: Seeding and layering of equatorial spread $\mathrm{F}$ by gravity waves, J. Geophys. Res., 95(A10), 17253-17260, 1990.

Hysell, D. L. and Kudeki, E.: Collisional shear instability in the equatorial F region ionosphere, Geophys. Res., 109, A11301, doi:10.1029/2004JA010636, 2004.

Kelley, M. C., Larsen, M. F., and LaHoz, C.: Gravity wave initiation of equatorial spread-F: A case study, J. Geophys. Res., 86, 90879100, 1981.

Keskinen, M. J., Ossakow, S. L., and Fejer, B. G.: Threedimensional nonlinear evolution of equatorial spread-F bubbles, Geophys. Res. Lett., 30(16), 1855, doi:10:1029/2003GL017418, 2003.

Kherani, E. A., Kelley, M. C., Carlos, F., and de Paula, E. R.: The effect of pressure gradient in the linear growth of equatorial Fregion Rayleigh-Taylor instability: An electromagnetic effect, J. Geophys. Res., in review, 2009b.

Kherani, E. A., Lognonne, P., Kamath, N., Crespon, F., and Garcia, R.: Response of the Ionosphere to the seismic trigerred acoustic wave: electron density and electromagnetic fluctuations, Geophys. J. Int., 176, 1-13, 2009c.

Kudeki, E. and Bhattacharyya, S.: Post sunset vortex in equatorial F-region plasma drifts and implications for bottom-side spread-F, J. Geophys. Res., 104, 28163-28170, 1999.

Kudeki, E., Akgiray, A., Milla, M., Chau, J. L., and Hysell, D. L.: Equatorial spread-F initiation: post-sunset vortex, thermospheric winds, gravity waves, J. Atmos. Solar-Terr. Phys., 69, 2416-2427, 2007.

Linson, I. M. and Workman, J. B.: Formation of striations in ionospheric plasma clouds, J. Geophys. Res., 75, 3211-3219, 1970.

MacDougall, J. W., Abdu, M. A., Jayachandran, P. T., Cecile, J.-F., and Batista, I. S.: Pre sunrise spread F at Fortaleza, J. Geophys. Res., 103(A10), 23415-23425, 1998.

Maruyama, T.: A diagnostic model for equatorial spread F 1. Model description and applicationto electric field and neutral wind effects, J. Geophys. Res., 93, 14611-4622, 1988.

McClure, J. P., Sing, S., Bamgboye, D. K., Johnson, F. S., and Hyusub Kil: Occurrence of equatorial F region irregularities: Evidence for tropospheric seeding, J. Geophys. Res., 103, 29119 
29135, 1998.

Prakash, S.: Production of electric filed perturbations by gravity wave winds in the $\mathrm{E}$ region suitable for initiating equatorial spread F, J. Geophys. Res., 104(A5), 10051-10069, 1999.

Rishbeth, H.: Polarization fields produced by winds in the equatorial F region, Planet. Space Sci., 19, 357-369, 1971.

Rottger, J.: Equatorial spread F by electric fields and atmospheric gravity waves generated by thunderstorms, J. Atmos. Terr. Phys., 43, 453-462, 1981.

Sastri, J. H., Abdu, M. A., Batista, I. S., and Sobral, J. H. A.: Onset conditions of equatorial (range) spread F at Fortaleza, Brazil, during the June solstice, J. Geophys. Res., 102(A11), 2401324021, 1997.

Sekar, R., Suhashini, R., and Raghavarao, R.: Effects of vertical winds and electric fields in the nonlinear evolution of equatorial spread F, J. Geophys. Res., 99, 2205-2213, 1994.
Scali, J. L., Reinisch, B. W., Heinselman, C. J., and Bullett, T.: Coordinated digisonde and incoherent scatter radar $\mathrm{F}$ region drift measurements at Sondre Stromfjord, Radio Sci., 30, 1481-1498, 1995.

Sultan, P. J.: Linear theory and modeling of the Rayleigh-Taylor instability leading to the occurrence of equatorial spread F, J. Geophys. Res., 101, 26875-26891, 1996.

Takahashi, H., Taylor, M. J., Pautet, P.-D., Medeiros, A. F., Gobbi, D., Wrasse, C. M., Fechine, J., Abdu, M. A., Batista, I. S., Paula, E., Sobral, J. H. A., Arruda, D., Vadas, S. L., Sabbas, F. S., and Fritts, D. C.: Simultaneous observation of ionospheric plasma bubbles and mesospheric gravity waves during the SpreadFEx Campaign, Ann. Geophys., 27, 1477-1487, 2009, http://www.ann-geophys.net/27/1477/2009/.

Zalesak, S. T., Ossakow, S. L., and Chaturvedi, P. K.: Nonlinear equatorial spread F: The effect of neutral winds and background Pedersen conductivity, J. Geophys. Res., 87, 151-166, 1982. 